\title{
Monitoring of Systemic Lupus Erythematosus Pregnancies: A Systematic Literature Review
}

\author{
Emily G. McDonald, Lyne Bissonette, Stephanie Ensworth, Natalie Dayan, Ann E. Clarke, \\ Stephanie Keeling, Sasha Bernatsky, and Evelyne Vinet
}

ABSTRACT. Objective. Few data exist to guide the frequency and type of monitoring in systemic lupus erythematosus (SLE) pregnancies. A systematic literature review was performed to address this gap in the literature.

Methods. A systematic review of original articles (1975-2015) was performed using Medline, Embase, and Cochrane Library. We included search terms for SLE, pregnancy, and monitoring. We also hand-searched reference lists, review articles, and grey literature for additional relevant articles. Results. The search yielded a total of 1106 articles. After removing 117 duplicates, 929 articles that were evidently unrelated to our topic based on title and/or abstract, and 7 that were in a language other than English or French, 53 articles were included for full-text review. Following a more in-depth review, 15 were excluded: 6 did not use any measure of SLE activity and 6 did not specifically address SLE monitoring in pregnancy; 1 case series, 1 review, and 1 metaanalysis were removed. Among the 38 included studies, presence of active disease, antiphospholipid (aPL) antibodies positivity, and abnormal uterine and umbilical artery Doppler studies predicted poor pregnancy outcomes. No studies evaluated an evidence-based approach to the frequency of monitoring.

Conclusion. Few existing studies address monitoring for optimal care during SLE pregnancies. The available data imply roles for aPL antibodies measurement (prior to pregnancy and/or during the first trimester), uterine and umbilical artery Doppler studies in the second trimester, and following disease activity. Optimal frequency of monitoring is not addressed in the existing literature. (First Release July 15 2018; J Rheumatol 2018;45:1477-90; doi:10.3899/jrheum.171023)

Key Indexing Terms:

SYSTEMIC LUPUS ERYTHEMATOSUS MONITORING

From the Division of General Internal Medicine, and the Division of Rheumatology, Department of Medicine, McGill University Health Centre, Montréal; Division of Rheumatology, Department of Medicine, University of Sherbrooke, Sherbrooke, Québec; Division of Rheumatology, Mary Pack Arthritis Center, University of British Columbia, Vancouver, British Columbia; Division of Rheumatology, Department of Medicine, University of Alberta, Edmonton; Division of Rheumatology, Department of Medicine, University of Calgary, Calgary, Alberta, Canada.

EV received salary funding from the Fonds de Recherches Santé Québec (FRSQ). EGM received funding from an FRSQ Master's award and the $M c$ Gill Clinician Investigator Program. SK received funding through a Canadian Institutes of Health Research Dissemination Grant.

E.G. McDonald, MD, MSc, Division of General Internal Medicine, Department of Medicine, McGill University Health Centre; L. Bissonette, MD, Division of Rheumatology, Department of Medicine, University of

Sherbrooke; S. Ensworth, MD, Assistant Professor, Division of Rheumatology, University of British Columbia; N. Dayan, MD, MSc, Division of General Internal Medicine, Department of Medicine, McGill University Health Centre; A.E. Clarke, MD, MSc, Division of

Rheumatology, Department of Medicine, University of Calgary;

S. Keeling, MD, MSc, Division of Rheumatology, Department of Medicine,

University of Alberta; S. Bernatsky, MD, PhD, Division of Rheumatology, Department of Medicine, McGill University Health Centre; E. Vinet, MD, PhD, Division of Rheumatology, Department of Medicine, McGill University Health Centre.

Address correspondence to Dr. E. Vinet, Assistant Professor, McGill University Health Centre, 5252 De Maisonneuve West, Montreal, Québec H4A3J1, Canada.E-mail: evelyne.vinet@mcgill.ca

Full Release Article. For details see Reprints and Permissions at jrheum.org Accepted for publication January 25, 2018.

\section{PREGNANCY \\ ADVERSE PREGNANCY OUTCOMES}

Systemic lupus erythematosus (SLE) predominantly affects women during their reproductive years, occurring in about $1 / 1000$ women aged between 15 and 45 years ${ }^{1}$. SLE is associated with substantial maternal and fetal morbidity during pregnancy. Compared with the non-SLE population, SLE has been shown to be associated with an increased risk of preterm birth, cesarean delivery, preeclampsia, low birth weight, intrauterine growth restriction (IUGR), congenital heart block (CHB), and intrauterine and neonatal death $2,3,4,5$. Preterm birth is the most common adverse pregnancy outcome in women with SLE, with incidence ranging from $15 \%$ to $50 \%$ (as opposed to $10 \%$ in unaffected women), with increased risk in women with lupus nephritis or high disease activity. In the general population, preterm birth is the leading cause of neonatal death and the second most common cause of death (after pneumonia) in children younger than 5 years ${ }^{6}$.

SLE has a waxing and waning course and it has been shown that if women conceive during a period of disease quiescence, this will minimize the risk of flare in pregnancy, but will not eliminate it, with rates of flare still ranging between $20 \%$ and $40 \%$ of pregnancies that are conceived during a period of remission $7,8,9,10$. In addition to a greater frequency of pregnancy complications, the SLE disease course itself can be negatively affected by pregnancy, with a

Personal non-commercial use only. The Journal of Rheumatology Copyright () 2018. All rights reserved. 
greater number of women developing an SLE flare in the peripartum period ${ }^{11,12,13}$.

SLE pregnancies are considered high risk, being associated with higher maternal and fetal morbidity. Although the majority of SLE pregnancies end with live births, active disease and major organ involvement can affect the outcomes in both mother and fetus. In addition, major fetal issues such as IUGR and neonatal SLE syndromes make monitoring imperative in SLE pregnancies ${ }^{14}$.

Finally, some symptoms of SLE can be silent, such as renal flare or thrombocytopenia, emphasizing the need for closer monitoring in pregnancy; these silent flares can still increase the chance of obstetrical complications ${ }^{15}$.

Quality indicators pertaining to reproductive health in SLE have been developed but are limited only to the recommendation that anti-SSA/SSB and antiphospholipid (aPL) antibodies be documented in the chart, prior to conception ${ }^{16}$. Optimal quality indicators remain undefined, and topics to be addressed include not only laboratory monitoring but also newer imaging modalities for monitoring high-risk pregnancies, such as umbilical and uterine artery Doppler studies. These are believed to be useful in monitoring pregnancies at high risk of placental insufficiency, which include SLE pregnancies ${ }^{17}$. Because the issue of how best to follow disease activity, and how often to monitor for disease flares, has not been systematically addressed in the literature, current practice is heterogeneous and not necessarily evidence-based ${ }^{18}$.

To address this important knowledge gap, a Canadian SLE working group was established, funded by the Canadian Institutes of Health Research and endorsed by the Canadian Rheumatology Association. One of the aims of this group was to determine what investigations are needed to optimally monitor pregnancy in SLE, in the Canadian context (which includes universal healthcare access).

\section{MATERIALS AND METHODS}

The systematic literature review was performed according to the Preferred Reporting Items for Systematic review and Meta-analysis Protocols (PRISMA-P) 2015 statement $^{19}$ (Table 1).

The review was conducted through 3 search engines: Embase, Medline, and Cochrane. The search was performed on publications between 1975 and 2015, without any language restrictions. We used Medical Subject Heading and free text terms adapted for each database to identify original articles. We included search terms for SLE, pregnancy, and monitoring variables. All terms within each set were combined using the Boolean operator "OR" and then the 3 sets were combined using "AND." This was supplemented by hand-searching reference lists, review articles, and grey literature for relevant articles not identified by the electronic searches, as well as including relevant articles published after completion of our review. Exclusion criteria then included any abstract in a language other than French or English, case reports, case series, and review articles.

Study selection. First, titles from the initial search were reviewed by 3 individuals (EGM, LB, and EV) to initially include any potential studies related to the study question, and to exclude any duplicates. Second, titles and abstracts were reviewed to identify relevant studies that met our inclusion criteria and to exclude case reports and studies unrelated to the systematic review. Third, 2 reviewers (EGM and LB) independently reviewed each full-text article for inclusion in the final set of articles, with a third reviewer (EV) settling discrepancies. Two reviewers (EGM and EV) summarized evidence on monitoring of SLE in pregnancy. None of the reviewers were blinded to the authors or journal titles.

Data extraction and quality assessment. Relevant data pertaining to monitoring were extracted from each article as well as general information such as country of the study, type of study, year of publication, and first author. Monitoring was divided into 4 categories: serological tests [which included anti-DNA, antiextractable nuclear antibody, IgG and IgM anticardiolipin antibodies (aCL), lupus anticoagulant (LAC), and complement levels]; measures of SLE activity using a validated scoring system [examples include the SLE Disease Activity Index (SLEDAI) and European Consensus Lupus Activity Measure scores; studies that did not use any type of scoring system to measure disease activity were not included]; obstetrical Doppler ultrasound monitoring (either uterine or umbilical artery Doppler studies); and other (monitoring variables not falling into one of the above categories). When applicable, we recorded whether the frequency of monitoring was addressed, as well as any associations with negative maternal or obstetrical outcomes. Maternal outcomes were classified as the development of preeclampsia, or worsening of SLE disease activity, including lupus nephritis. Obstetrical outcomes were classified as IUGR, spontaneous abortion (prior to 20 weeks of gestational age), small for gestational age (SGA) or low birth weight (SGA and low birth weight were defined within individual studies), preterm delivery (prior to 37 weeks gestational age), complete $\mathrm{CHB}$, or intrauterine fetal demise. Relevant data were extracted, synthesized, and presented in tabular format.

Risk of bias in individual studies: the Newcastle-Ottawa scale. Final studies included in the systematic review were evaluated for quality of evidence and risk of bias using the Newcastle-Ottawa scale, which uses a star system whereby a study is judged on 3 measures: the selection of the study groups, the comparability of the groups, and how the exposure for case-control (or outcome of interest for cohort studies) was ascertained.

\section{RESULTS}

Study characteristics. A general description of the characteristics of each study is presented in Table $2^{2,9,13,15,20-53}$ including first author, year of publication, study population, and study type, as well as monitoring variables studied. A description of the search process, including the reasons for excluded studies, is shown in Figure 1. In total, 1106 titles were evaluated from the initial search strategy, of which 117 were removed because they were duplicates, and 7 were removed because they were in a language other than French or English. Then, on initial review of the title and/or abstracts, 929 articles that did not address SLE monitoring in pregnancy and were removed, leaving 53 articles that were reviewed in depth. Upon full review of the 53 articles, it was found that 6 more did not address SLE monitoring in pregnancy, 6 did not use any scoring system for SLE disease activity, 1 was a review, 1 was a case series, and 1 was a metaanalysis. These were removed, leaving 38 original observational studies for review. Final studies were selected based on reporting of adverse obstetrical or maternal outcomes related to monitoring variables (Figure 1) in SLE. Participant characteristics. The majority of studies evaluated monitoring of SLE during pregnancy and immediately postpartum. Only a few studies looked specifically at lupus nephritis or focused solely on pregnancies in mothers who were anti-Ro- and/or La-antibody positive. The study populations were multiethnic, with patients from across the world

Personal non-commercial use only. The Journal of Rheumatology Copyright $\odot$ (2018. All rights reserved 
Table 1. PRISMA-P checklist for preferred reporting of systematic reviews.

\begin{tabular}{|c|c|c|}
\hline Section and Topic & Item No. & Checklist Item \\
\hline \multicolumn{3}{|c|}{ Administrative information } \\
\hline \multicolumn{3}{|l|}{ Title } \\
\hline Update & $1 b$ & $\begin{array}{l}\text { If the protocol is for an update of a previous systematic review, identify as }- \text { This is not an update of a previous } \\
\text { protocol for a systematic review. }\end{array}$ \\
\hline Registration & 2 & $\begin{array}{l}\text { If registered, provide the name of the registry (such as PROSPERO) and registration number - This systematic review } \\
\text { was not registered. }\end{array}$ \\
\hline Contact & $3 a$ & $\begin{array}{l}\text { Provide name, institutional affiliation, e-mail address of all protocol authors; provide physical mailing address of } \\
\text { corresponding author - Emily Gibson McDonald }{ }^{1} \text {, Royal Victoria Hospital, } 1001 \text { Decarie St., Montreal, Quebec } \\
\text { H4A 3J1, Canada. Evelyne Vinet }{ }^{1} \text {, Stephanie Keeling }{ }^{2} \text {, Natalie Dayan }{ }^{1} \text {, Lyne Bissonette }{ }^{3} \text {, Sasha Bernatsky }{ }^{1} \text {, Stephanie } \\
\text { Ensworth }{ }^{4} \text {, Ann E. Clarke }{ }^{5} .{ }^{1} \text { McGill University Health Centre, }{ }^{2} \text { University of Alberta, }{ }^{3} \text { University of Laval, }{ }^{4} \text { Mary } \\
\text { Pack Arthritis Centre, }{ }^{5} \text { University of Calgary. }\end{array}$ \\
\hline Contributions & $3 b$ & $\begin{array}{l}\text { Describe contributions of protocol authors and identify the guarantor of the review - Emily McDonald and Evelyne } \\
\text { Vinet were involved in reading the abstracts, excluding unrelated abstracts, drafting the manuscript including reading } \\
\text { articles in full and collating the data. Lyne Bissonette read and excluded unrelated abstracts. Natalie Dayan was } \\
\text { involved in the supervision of Lyne Bissonette. Evelyne Vinet developed the PICO question and the search strategy } \\
\text { and supervised Emily McDonald. Stephanie Keeling provided content expertise and supervision of the GRADE recom- } \\
\text { mendations. Stephanie Ensworth and Ann E. Clark are part of the Canadian SLE Working Group. Evelyne Vinet is } \\
\text { the guarantor of the manuscript. }\end{array}$ \\
\hline Amendments & 4 & $\begin{array}{l}\text { If the protocol represents an amendment of a previously completed or published protocol, identify as such and list } \\
\text { changes; otherwise, state plan for documenting important protocol amendments - This manuscript does not represent } \\
\text { a protocol amendment. }\end{array}$ \\
\hline
\end{tabular}

$\begin{array}{ll}\begin{array}{l}\text { Support } \\ \text { Sources }\end{array} & 5 \mathrm{a} \\ & \\ & \\ \text { Sponsor } & 5 \mathrm{~b} \\ \text { Role of sponsor } & 5 \mathrm{c} \\ \quad \text { or funder } & \\ \text { Introduction } & \\ \text { Rationale } & 6\end{array}$

Indicate sources of financial or other support for the review - EV received salary funding from the Fonds de Recherches Santé Québec (FRSQ). EGM received funding from an FRSQ Master's award and the McGill Clinician Investigator Program. SK received funding through a CIHR Dissemination Event. This work will be used by the Canadian SLE Working Group to develop recommendations for the diagnosis and monitoring of SLE.

b Provide name for the review funder and/or sponsor - The review was not funded.

Describe roles of funder(s), sponsor(s), and/or institution(s), if any, in developing the protocol - Cochrane Review was involved in the development of the PICO question.

Describe the rationale for the review in the context of what is already known - Pregnant patients with SLE have higher rates of adverse obstetrical outcomes and SLE has a high likelihood of flaring in pregnancy, which in term can lead to worse obstetrical outcomes; there exist few guidelines for the monitoring of pregnancy in SLE; it is known that quiescent disease for several months prior to conception is associated with improved outcomes. It is not known what should be monitored throughout pregnancy in terms of blood and urine tests, serologies, ultrasounds, and disease activity and at what frequency.

Objectives 7 Provide an explicit statement of the question(s) the review will address with reference to participants, interventions, comparators, and outcomes (PICO).

$\mathrm{P}$ - pregnant SLE patients including all women of child-bearing age.

I - laboratory investigations: antiphospholipid antibodies, Ro/SSA and La/SSB antibodies, anti-DNA antibody, complement levels, ESR, CRP, uric acid; renal function: disease activity, SLEPDAI, SLEDAI, BILAG, SLAM, ECLAM, SRI, CBC differential, lupus activity index, DNA antibodies; other: SLICC, monitoring of the baby, blood pressure.

$\mathrm{C}-$ frequency.

$\mathrm{O}$ - Preterm birth, preeclampsia/eclampsia, stillbirth, small for gestational age, intrauterine growth restriction, congenital heart block, fetal heart block, neonatal lupus, stillbirth, miscarriage, SLE flare; gestational hypertension: renal insufficiency, maternal death, thromboembolism event, premature rupture of membranes, premature labor/delivery; secondary: gestational diabetes, cesarean delivery.

Methods: For the following section please refer to the Materials and Methods section of the manuscript

Eligibility criteria $8 \quad$ Specify the study characteristics (such as PICO, study design, setting, time frame) and report characteristics (such as years considered, language, publication status) to be used as criteria for eligibility for the review.

Information sources 9 Describe all intended information sources (such as electronic databases, contact with study authors, trial registers, or other grey literature sources) with planned dates of coverage.

Search strategy $10 \quad \begin{aligned} & \text { Present draft of search strategy to be used for at least } 1 \text { electronic database, including planned limits, such that it could } \\ & \text { be repeated. }\end{aligned}$

Study records

Data management 11a Describe the mechanism(s) that will be used to manage records and data throughout the review - All records were managed in an Excel spreadsheet that included author, year, population, exposure, outcomes, and results. 
Table 1. Continued.

\begin{tabular}{|c|c|c|}
\hline Section and Topic & Item No. & Checklist Item \\
\hline Selection process & $11 \mathrm{~b}$ & $\begin{array}{l}\text { State the process that will be used for selecting studies (such as } 2 \text { independent reviewers) through each phase of the } \\
\text { review (that is, screening, eligibility, and inclusion in metaanalysis) - Three authors were involved in screening the } \\
\text { abstracts [ } 2 \text { authors initially - EM and LB, and a third author (EV) was involved if the first } 2 \text { authors did not agree } \\
\text { on the eligibility of a study]. Two authors read the articles included for in-depth review and decided on the final exclu- } \\
\text { sions (EM and EV). }\end{array}$ \\
\hline Data collection process & $\mathrm{s} \quad 11 \mathrm{c}$ & $\begin{array}{l}\text { Describe planned method of extracting data from reports (such as piloting forms, done independently, in duplicate), } \\
\text { any processes for obtaining and confirming data from investigators - Two authors reviewed the articles included for } \\
\text { the final review and based on an Excel spreadsheet extracted the data and collated it. }\end{array}$ \\
\hline Data items & 12 & $\begin{array}{l}\text { List and define all variables for which data will be sought (such as PICO items, funding sources), any pre-planned } \\
\text { data assumptions and simplifications - For variables please refer to the in-depth description of the PICO question } \\
\text { above where each component of the I and } O \text { are described in detail. }\end{array}$ \\
\hline $\begin{array}{r}\text { Outcomes and } \\
\text { prioritization }\end{array}$ & 13 & $\begin{array}{l}\text { List and define all outcomes for which data will be sought, including prioritization of main and additional outcomes, } \\
\text { with rationale - Refer to the PICO question above where all elements of the outcomes are described. }\end{array}$ \\
\hline $\begin{array}{l}\text { Risk of bias in } \\
\text { individual studies }\end{array}$ & 14 & $\begin{array}{l}\text { Describe anticipated methods for assessing risk of bias of individual studies, including whether this will be done } \\
\text { at the outcome or study level, or both; state how this information will be used in data synthesis - Studies were } \\
\text { evaluated for quality using the Newcastle-Ottawa Scale (described in depth in the body of the thesis). }\end{array}$ \\
\hline \multirow{3}{*}{ Data synthesis } & $15 b$ & $\begin{array}{l}\text { If data are appropriate for quantitative synthesis, describe planned summary measures, methods of handling data, and } \\
\text { methods of combining data from studies, including any planned exploration of consistency (such as } \mathrm{I}^{2}, \text { Kendall's } \tau \text { ) } \\
\text { - Not applicable for this systematic review (relevant for metaanalysis only). }\end{array}$ \\
\hline & $15 \mathrm{c}$ & $\begin{array}{l}\text { Describe any proposed additional analyses (such as sensitivity or subgroup analyses, metaregression) - Not applicable } \\
\text { for this systematic review (relevant for metaanalysis only). }\end{array}$ \\
\hline & $15 \mathrm{~d}$ & $\begin{array}{l}\text { If quantitative synthesis is not appropriate, describe the type of summary planned - Not applicable for this systematic } \\
\text { review (relevant for metaanalysis only). }\end{array}$ \\
\hline Metabias(es) & 16 & $\begin{array}{l}\text { Specify any planned assessment of metabias(es), such as publication bias across studies, selective reporting within } \\
\text { studies - Not applicable for this systematic review (relevant for metaanalysis only). }\end{array}$ \\
\hline $\begin{array}{l}\text { Confidence in cumulative } \\
\text { evidence }\end{array}$ & e 17 & $\begin{array}{l}\text { Describe how the strength of the body of evidence will be assessed (such as GRADE) - This is described in great } \\
\text { detail in the methods section of the thesis. }\end{array}$ \\
\hline
\end{tabular}

PRISMA-P: Preferred Reporting Items for Systematic Review and Metaanalysis Protocols; GRADE: Grading of Recommendations Assessment, Development, and Evaluation; SLE: systemic lupus erythematosus; CIHR: Canadian Institutes of Health Research; PICO search strategy: Participants - Intervention Comparison/Comparator - Outcome; ESR: erythrocyte sedimentation rate; CRP: C-reactive protein; SLEPDAI: SLE in Pregnancy Disease Activity Index; SLEDAI: Systemic Lupus Erythematosus Disease Activity Index; BILAG: British Isles Lupus Assessment Group index; SLAM: Systemic Lupus Activity Measure; ECLAM: European Consensus Lupus Activity Measure; SRI: SLEDAI-2K Responder Index 50; CBC: complete blood count; SLICC: Systemic Lupus International Collaborating Clinics.

including North America, Europe, Latin America, Asia, and the Middle East.

Monitoring variables characteristics. Of 38 final articles evaluated, 22/38 (58\%) addressed the value of measuring serology, including the measurement of aPL antibodies prior to pregnancy and/or during the first trimester, and 18/38 (47\%) assessed monitoring for SLE flare with a validated scoring system, such as the SLEDAI (Figure 2). The utility of umbilical and/or uterine artery Doppler monitoring for predicting poor obstetrical outcomes was evaluated in 5/38 $(13 \%)$ articles. Disease activity scoring systems modified to account for the physiologic changes of pregnancy were observed in 4 articles, such as the SLE in Pregnancy Disease Activity Index ${ }^{54}$ and the Lupus Activity Index in Pregnancy ${ }^{55}$. Of note, none of them have been formally validated.

Outcomes. Findings from each study are summarized and presented in Table $3^{2,9,13,15,20-53}$; studies were evaluated for risk of bias using the Newcastle-Ottawa scale and the quality of data is presented in Table $42,9,13,15,20-53$. Across the studies included for full review, the presence of active disease (measured by various scoring systems), aPL antibodies positivity, and abnormal uterine and umbilical artery Doppler studies predicted poor pregnancy outcomes. Low complement and thrombocytopenia at the beginning of pregnancy were also predictors of poor obstetrical outcomes. No studies that assessed the value of serological and disease activity monitoring evaluated an evidence-based approach to the frequency of monitoring. Studies that addressed uterine and umbilical artery Doppler monitoring discussed the frequency at which these tests were performed but did not address the evidence behind the practice.

\section{DISCUSSION}

Summary of findings. In this systematic review examining monitoring in SLE pregnancies, 38 articles were included, after an initial screening of 1106 abstracts and 53 articles that were reviewed in depth. Final articles addressed the monitoring of SLE-related pregnancy and focused on 3 main 
Table 2. Study characteristics.

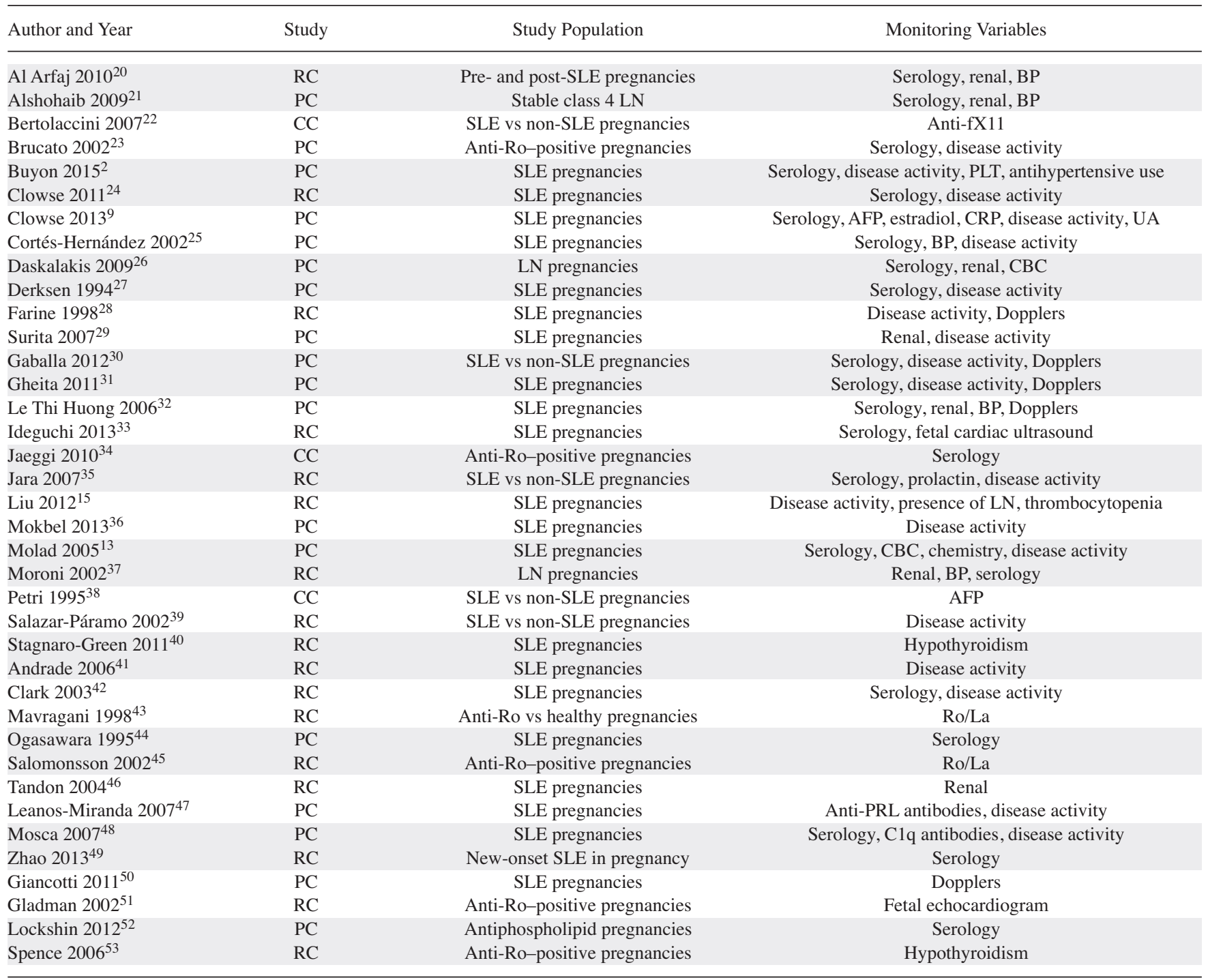

Renal = serum creatinine, urinalysis, urine microscopy, and/or urine protein-to-creatinine ratio. RC: retrospective cohort; PC: prospective cohort; CC: case control; SLE: systemic lupus erythematosus; PRL: prolactin; BP: blood pressure; PLT: platelets; AFP: alpha fetal protein; CRP: C-reactive protein; LN: lupus nephritis; UA: uric acid; CBC: complete blood count.

types of monitoring (blood and urine tests, SLE disease activity monitoring, and uterine or umbilical Doppler studies), as well as other types of monitoring. There have been no randomized controlled studies on this topic and so all articles were observational studies. The majority of studies were cohort studies that investigated predictors of poor maternal and fetal outcomes related to measures of SLE activity (both disease-specific and otherwise) throughout pregnancy. Studies included SLE women with varying degrees of disease activity, with some including only women with inactive or mildly active SLE, which might have influenced their findings.

Possible recommendations. Based on our systematic review of the literature, there may be a role for monitoring aPL (LAC and aCL), dsDNA, complement, and anti-Ro and anti-La antibodies prior to conception and early in pregnancy. Complement and dsDNA are likely helpful measures when a flare is suspected because these tests were shown in numerous studies that we examined to be predictive of poor outcomes $(20 / 37$ or $54 \%$ of studies addressed the predictive value of 1 or more of these monitoring variables). Because patients with quiescent SLE (i.e., with no or low disease activity) have fewer negative outcomes in pregnancy, if these autoantibodies and blood tests are measured prior to conception, it might help physicians stratify pregnancy risk in pregnant women and/or women planning a pregnancy. However, dsDNA, for example, does not always correlate with disease flare and/or activity in all patients and so this is

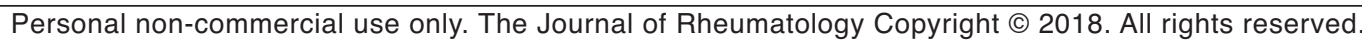




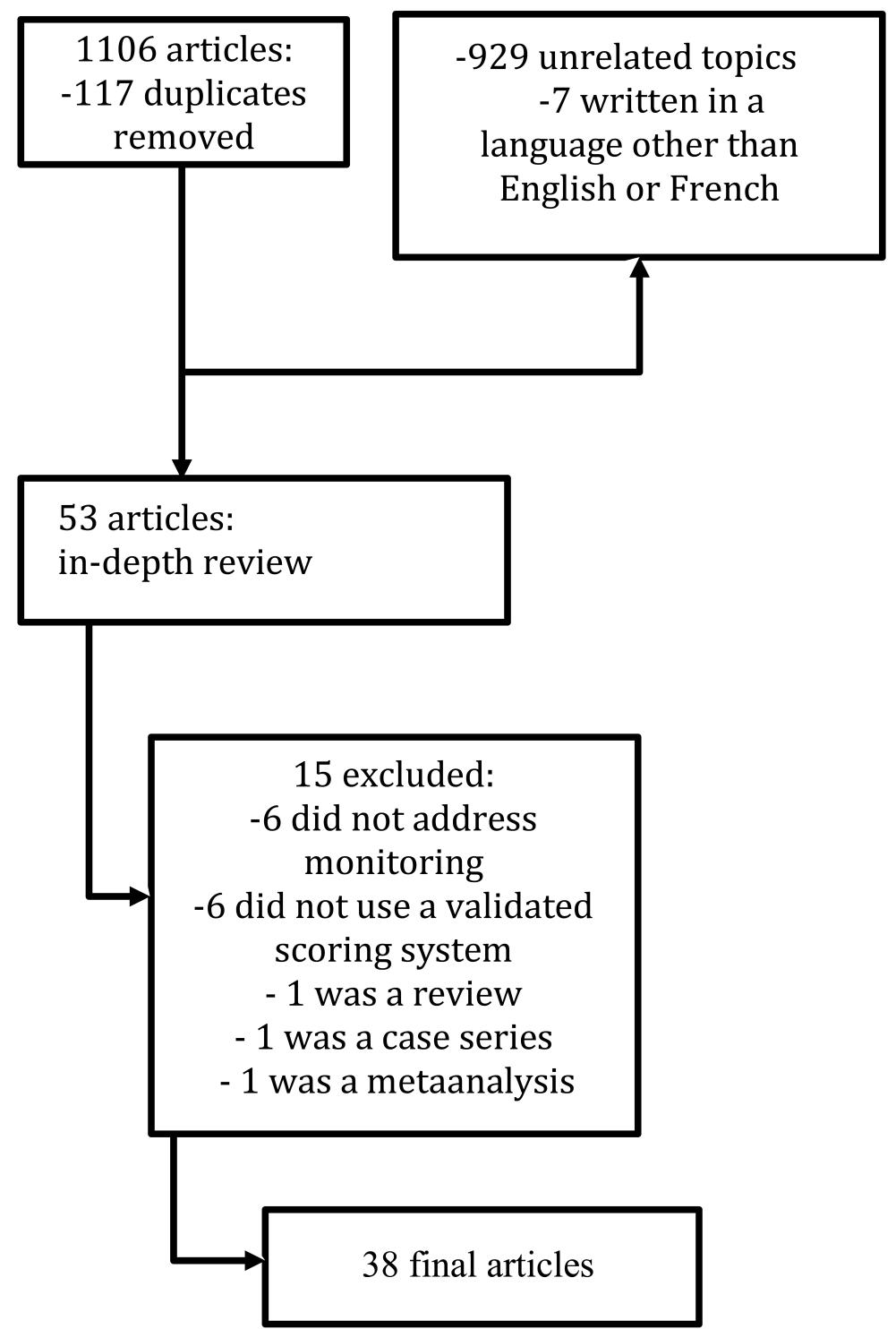

Figure 1. Flowchart of the article selection process.

not universally true, but might still contribute useful information. In addition, complement levels normally increase during pregnancy, which might further complicate its role as a reliable disease activity marker ${ }^{27}$.

The available literature also suggests that laboratory testing should be combined with evidence of increased disease activity index as measured by a validated scoring system, such as the SLEDAI, which has also shown to be associated with poor maternal and obstetrical outcomes in numerous studies included in this systematic review (18/38 or $47 \%$ ). However, no study has directly compared the predictive value of laboratory or clinical data alone as compared to SLEDAI (which combines clinical activity as well as laboratory data).
One expanding area of monitoring in SLE pregnancies relates to the role of uterine and umbilical Doppler studies. Absent end-diastolic velocities of the umbilical artery predict early pregnancy-induced hypertension/preeclampsia and fetal or neonatal death in non-SLE pregnancies ${ }^{56,57}$. Abnormalities demonstrating increased resistive indices, notching of the arteries, or in very severe cases, reversal of end-diastolic flow, are highly predictive of poor fetal outcomes in SLE pregnancies ${ }^{28,32}$. This type of monitoring is minimally invasive and could be considered in the second and third trimester if the expertise exists in the center where the patient's pregnancy is followed. This monitoring could therefore be used at the time of a suspected flare, or at the discretion of the maternal-fetal 


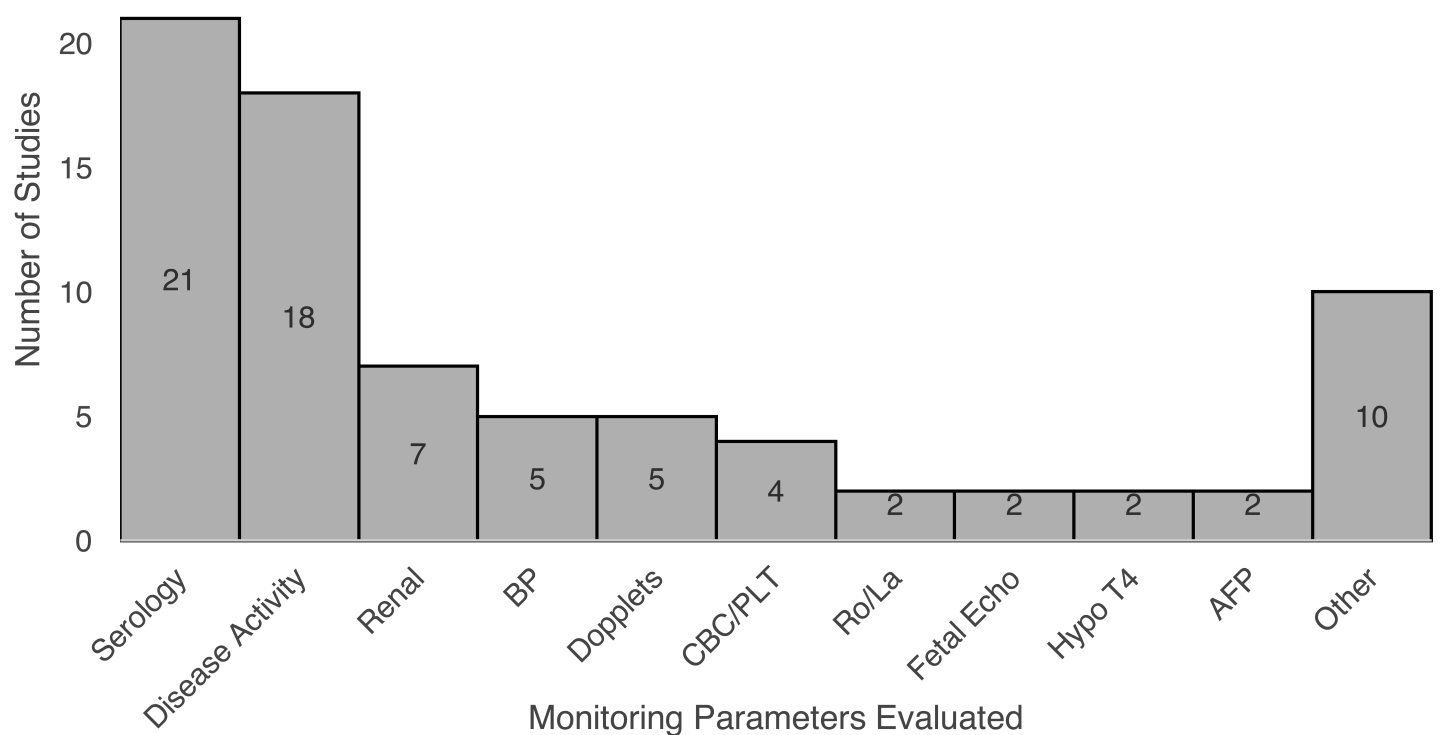

Figure 2. Histogram of the frequency of monitoring variables evaluated in the studies included in the systematic literature review. Serology = anti-DNA, antiextractable nuclear antibody, IgG and IgM anticardiolipin antibodies, lupus anticoagulant, and/or complement levels. Disease activity measured using a validated scoring system (examples include the SLEDAI and ECLAM scores; studies that did not use any type of scoring system to measure disease activity were not included). Renal = serum creatinine, urinalysis, urine microscopy and/or urine creatinine-to-protein ratio. Other = variables evaluated by only 1 included study (e.g., antifactor X11, C1q, and anti-PRL antibodies). AFP: alpha fetal protein; Hypo T4: hypothyroidism; CBC: complete blood count; PLT: platelets; BP: blood pressure; SLEDAI: Systemic Lupus Erythematosus Disease Activity Index; ECLAM: European Consensus Lupus Activity Measure; PRL: prolactin.

medicine or obstetrical medicine specialist who is involved in the patient's care.

Four studies looked at alternative or novel tests for predicting poor outcomes in pregnancy such as anti-C1q antibodies, antiprolactin antibodies, antifactor X11, and alpha-fetal protein. Given that these studies have not been reproduced, and many of these serological tests are not widely available, we would not recommend their routine use at this time for monitoring of SLE pregnancies.

Regarding other tests, it is reasonable to measure thyroid stimulating hormone levels early in pregnancy, because hypothyroidism was found to be associated with an increased risk of prematurity in pregnant women from the general population as well as pregnant women with SLE, and was associated with an increased incidence of complete CHB among the offspring of mothers with anti-Ro antibodies ${ }^{40,53}$.

Prior to our study and the meeting of the Canadian SLE Working Group, guidelines did not exist regarding what monitoring variables should be measured in SLE pregnancies and at what frequency. A systematic review of the literature revealed there is no evidence to guide the frequency of routine monitoring of complete blood count, chemistry profile including liver enzymes, and urinalysis (routine or microscopy) or urine protein to creatinine levels, during pregnancy with SLE. There is some evidence for a role for measuring anti-Ro and anti-La antibodies prior to conception and early in SLE-related pregnancy, to risk-stratify pregnancies regarding the development of complete CHB. Similarly, the presence of aPL is predictive of poor outcomes, particularly preeclampsia and fetal loss, and so may be reasonable to measure prior to conception or early in pregnancy. Monitoring SLE disease activity should be done, potentially with a validated scoring system such as the SLEDAI because it relies on assessment of different clinical aspects of the disease (i.e., clinical manifestations and specific blood tests). If a flare is suspected, measuring antidsDNA antibodies and complement levels may be helpful. Women with SLE should be considered for referral to an expert in maternal-fetal medicine for fetal monitoring with umbilical artery Doppler studies, given that Doppler abnormalities are predictive of adverse outcomes; among pregnant populations without SLE, this monitoring modality has improved fetal outcomes. This systematic review will inform guidelines for the type of and frequency of monitoring of SLE in pregnancy, which are being developed by the Canadian SLE Working Group.

Personal non-commercial use only. The Journal of Rheumatology Copyright @ 2018 . All rights reserved. 


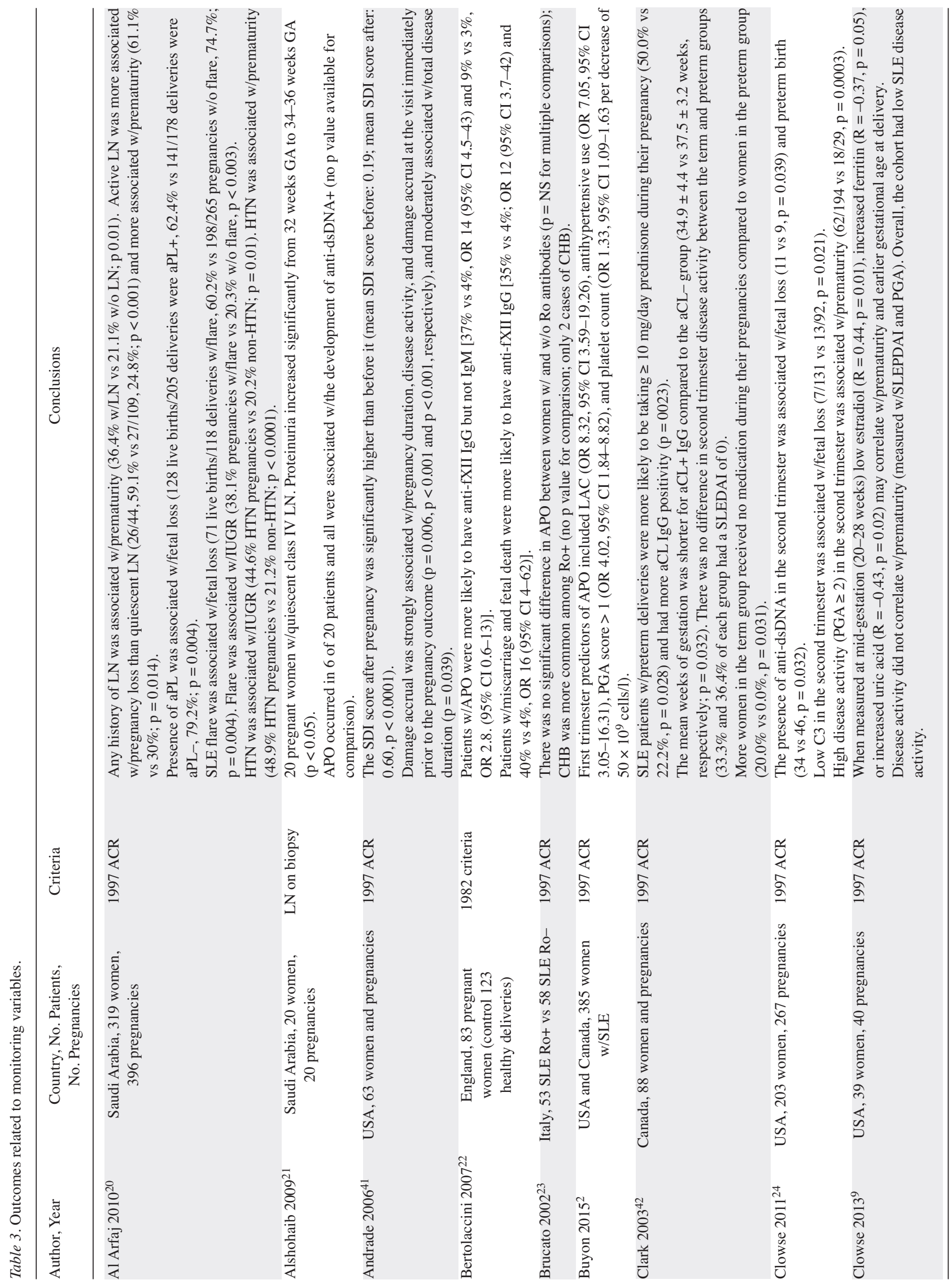

Personal non-commercial use only. The Journal of Rheumatology Copyright @ 2018 . All rights reserved. 


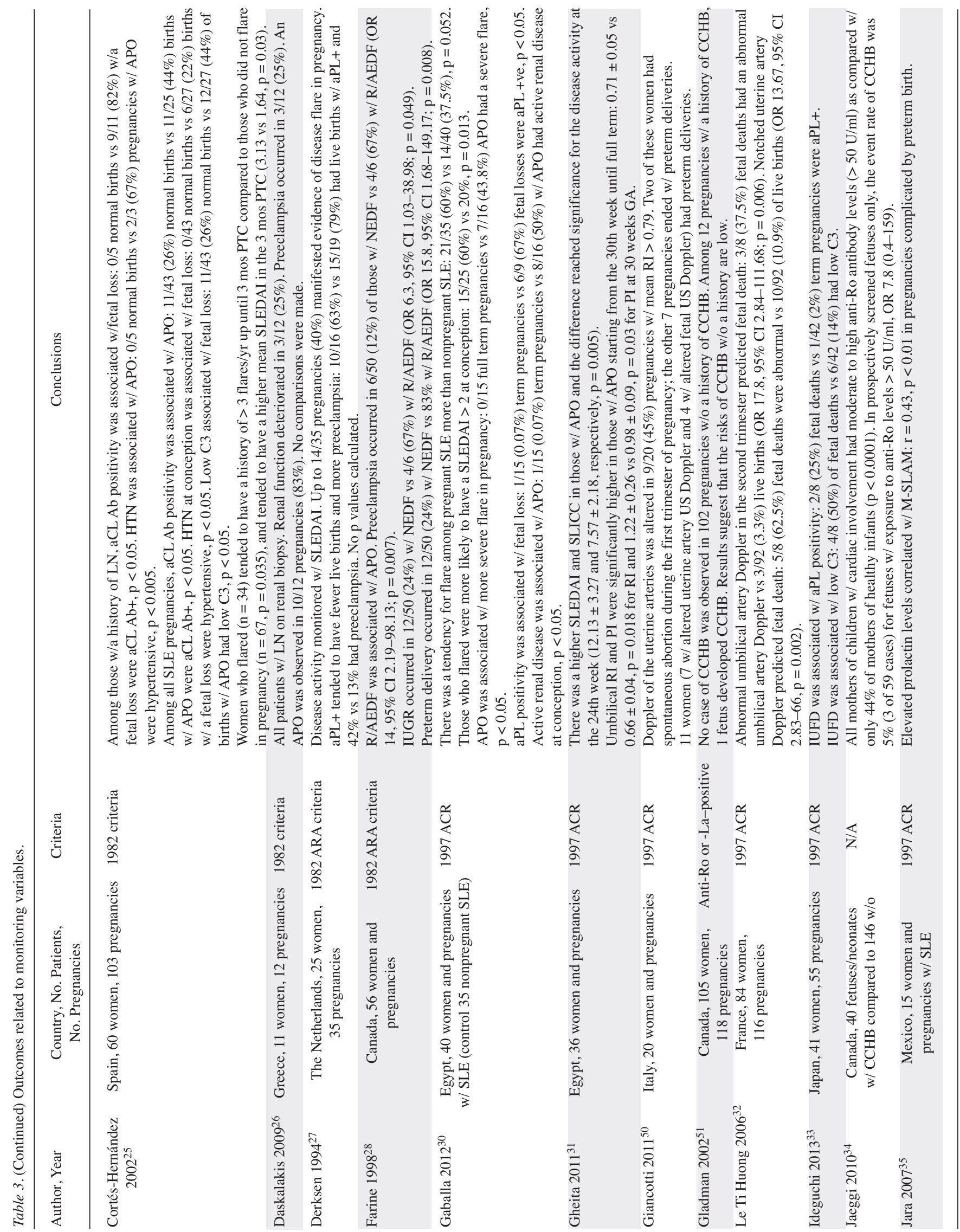

Personal non-commercial use only. The Journal of Rheumatology Copyright @ 2018 . All rights reserved. 


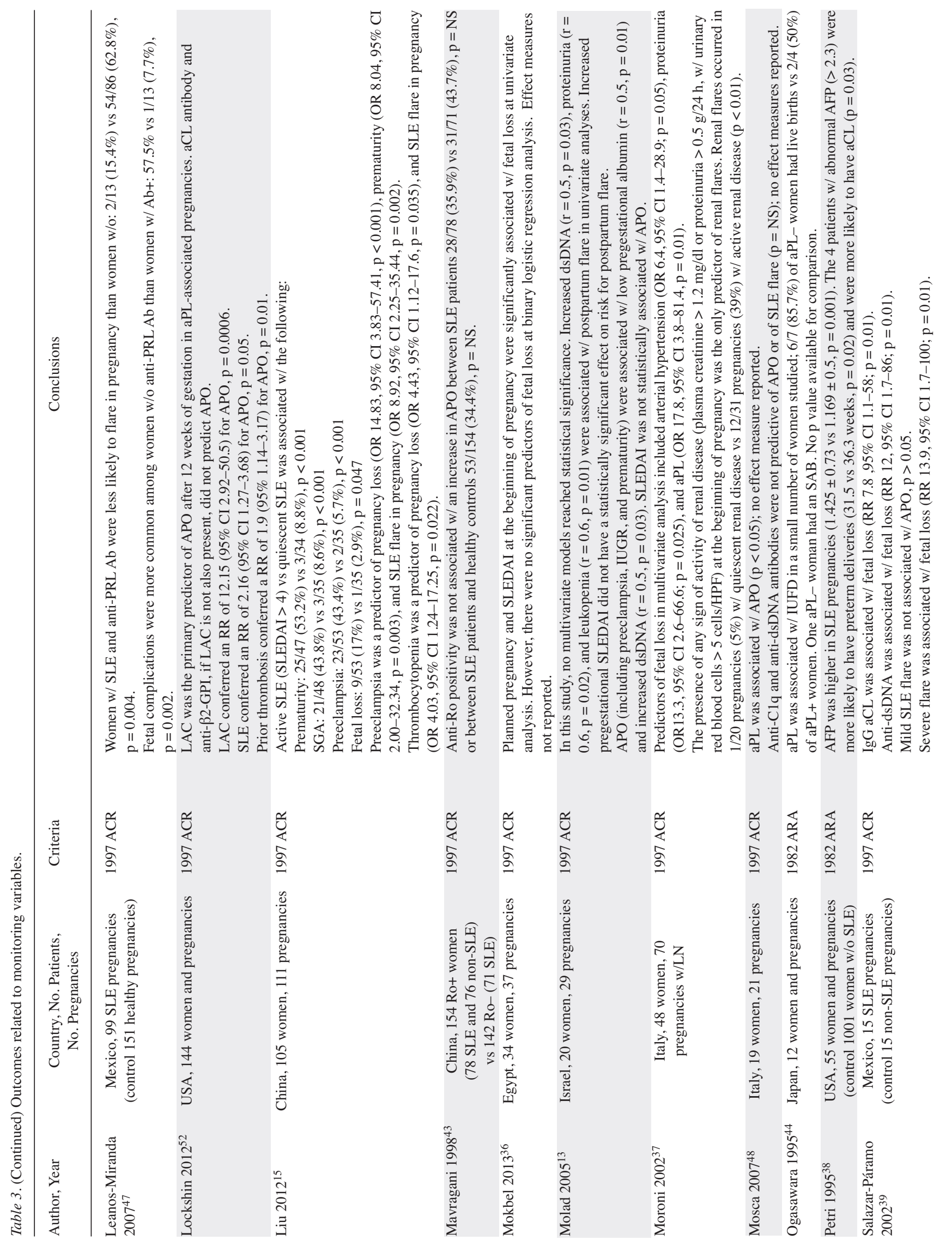

Personal non-commercial use only. The Journal of Rheumatology Copyright (c) 2018. All rights reserved. 


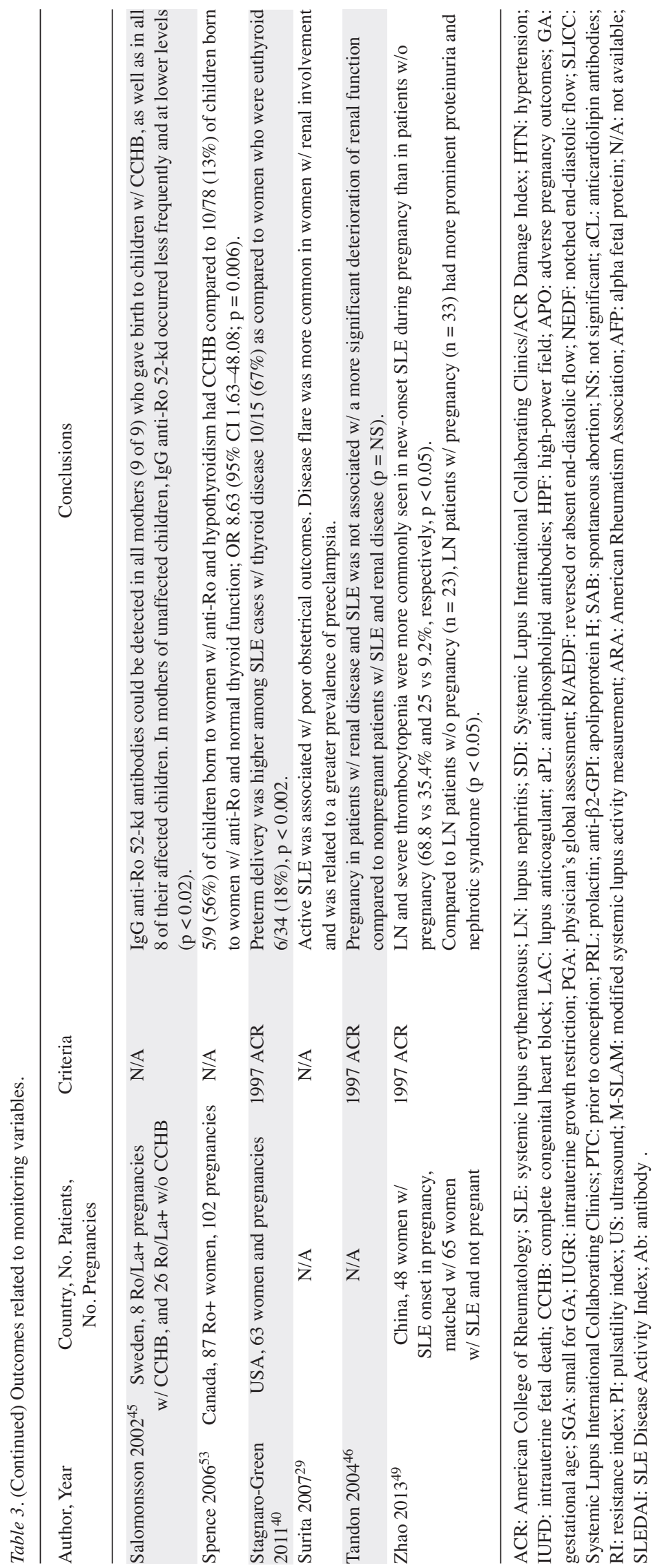

Personal non-commercial use only. The Journal of Rheumatology Copyright (c) 2018. All rights reserved. 
Table 4. Quality assessment by Newcastle-Ottawa scale.

\begin{tabular}{|c|c|c|c|c|}
\hline Author and Year & Selection $(/ 4)$ & Comparability (/2) & Outcome (/3) & Total (/9) \\
\hline Al Arfaj $2010^{20}$ & 4 & 2 & 3 & 9 \\
\hline Alshohaib $2009^{21}$ & 2 & 1 & 2 & 5 \\
\hline Brucato $2002^{23}$ & 3 & 1 & 3 & 7 \\
\hline Buyon $2015^{2}$ & 4 & 2 & 3 & 9 \\
\hline Clowse $2011^{24}$ & 3 & 1 & 3 & 7 \\
\hline Clowse $2013^{9}$ & 3 & 1 & 2 & 6 \\
\hline Cortés-Hernández $2002^{25}$ & 1 & 1 & 3 & 7 \\
\hline Daskalakis $2009^{26}$ & 3 & 1 & 3 & 5 \\
\hline Derksen $1994^{27}$ & 3 & 1 & 3 & 6 \\
\hline Farine $1998^{28}$ & 3 & 1 & 3 & 7 \\
\hline Surita $2007^{29}$ & 3 & 1 & 3 & 6 \\
\hline Gaballa $2012^{30}$ & 3 & 2 & 1 & 6 \\
\hline Gheita $2011^{31}$ & 3 & 1 & 1 & 5 \\
\hline Le Ti Huong $2006^{32}$ & 2 & 1 & 2 & 5 \\
\hline Ideguchi $2013^{33}$ & 3 & 1 & 2 & 6 \\
\hline Jara $2007^{35}$ & 2 & 1 & 1 & 4 \\
\hline Liu $2012^{15}$ & 3 & 2 & 2 & 7 \\
\hline Mokbel $2013^{36}$ & 3 & 2 & 2 & 7 \\
\hline Molad $2005^{13}$ & 3 & 1 & 2 & 6 \\
\hline Moroni $2002^{37}$ & 2 & 1 & 2 & 5 \\
\hline Salazar-Páramo $2002^{39}$ & 2 & 1 & 2 & 5 \\
\hline Stagnaro-Green $2011^{40}$ & 3 & 1 & 3 & 7 \\
\hline Andrade $2006^{41}$ & 3 & 2 & 2 & 7 \\
\hline Clark $2003^{42}$ & 3 & 1 & 2 & 6 \\
\hline Mavragani $1998^{43}$ & 3 & 2 & 2 & 7 \\
\hline Ogasawara $1995^{44}$ & 3 & 1 & 2 & 6 \\
\hline Tandon $2004^{46}$ & 3 & 2 & 3 & 8 \\
\hline Leanos-Miranda $2007^{47}$ & 2 & 1 & 2 & 5 \\
\hline Mosca $2007^{48}$ & 3 & 0 & 2 & 5 \\
\hline Giancotti $2011^{50}$ & 3 & 1 & 3 & 7 \\
\hline Gladman $2002^{51}$ & 3 & 1 & 3 & 7 \\
\hline Lockshin $2012^{52}$ & 4 & 2 & 3 & 9 \\
\hline Spence $2006^{53}$ & 1 & 1 & 3 & 5 \\
\hline Bertolaccini $2007^{22}$ & 1 & 1 & 2 & 4 \\
\hline Jaeggi $2010^{34}$ & 3 & 1 & 3 & 7 \\
\hline Petri $1995^{38}$ & 3 & 2 & 3 & 8 \\
\hline Salomonsson $2002^{45}$ & 3 & 1 & 3 & 7 \\
\hline Zhao $2013^{49}$ & 4 & 1 & 3 & 8 \\
\hline
\end{tabular}

\section{REFERENCES}

1. Fessel WJ. Epidemiology of systemic lupus erythematosus. Rheum Dis Clin North Am 1988;14:15-23.

2. Buyon JP, Kim MY, Guerra MM, Laskin CA, Petri M, Lockshin $\mathrm{MD}$, et al. Predictors of pregnancy outcomes in patients with lupus: a cohort study. Ann Intern Med 2015;163:153-63.

3. Wallenius M, Salvesen KA, Daltveit AK, Skomsvoll JF. Systemic lupus erythematosus and outcomes in first and subsequent births based on data from a national birth registry. Arthritis Care Res 2014;66:1718-24.

4. Clowse ME, Jamison M, Myers E, James AH. A national study of the complications of lupus in pregnancy. Am J Obstet Gynecol 2008;199:127.e121-6.

5. Lockshin MD, Sammaritano LR. Lupus pregnancy. Autoimmunity 2003;36:33-40.

6. World Health Organization. Preterm birth. [Internet. Accessed March 15, 2018.] Available from: www.who.int/mediacentre/factsheets/fs363/en/

7. Zhang W, Chen SL. An overview on systemic lupus erythematosus pregnancy. Mod Rheumatol 2003;13:293-300.
8. Koh JH, Ko HS, Kwok SK, Ju JH, Park SH. Hydroxychloroquine and pregnancy on lupus flares in Korean patients with systemic lupus erythematosus. Lupus 2015;24:210-17.

9. Clowse ME, Wallace DJ, Weisman M, James A, Criscione-Schreiber LG, Pisetsky DS. Predictors of preterm birth in patients with mild systemic lupus erythematosus. Ann Rheum Dis 2013;72:1536-9.

10. Ko HS, Ahn HY, Jang DG, Choi SK, Park YG, Park IY, et al. Pregnancy outcomes and appropriate timing of pregnancy in 183 pregnancies in Korean patients with SLE. Int J Med Sci 2011; 8:577-83.

11. Singh AG, Chowdhary VR. Pregnancy-related issues in women with systemic lupus erythematosus. Int J Rheum D 2015;18:171-81.

12. Yasmeen S, Wilkins EE, Field NT, Sheikh RA, Gilbert WM. Pregnancy outcomes in women with systemic lupus erythematosus. J Matern Fetal Med 2001;10:91-6.

13. Molad Y, Borkowski T, Monselise A, Ben-Haroush A, Sulkes J, Hod $\mathrm{M}$, et al. Maternal and fetal outcome of lupus pregnancy: A prospective study of 29 pregnancies. Lupus 2005;14:145-51.

14. Lateef A, Petri M. Managing lupus patients during pregnancy. Best practice \& research. J Clin Rheumatol 2013;27:435-47. 
15. Liu J, Zhao Y, Song Y, Zhang W, Bian X, Yang J, et al. Pregnancy in women with systemic lupus erythematosus: a retrospective study of 111 pregnancies in Chinese women. J Matern Fetal Neonatal Med 2012;25:261-6.

16. Gillis JZ, Panopalis P, Schmajuk G, Ramsey-Goldman R, Yazdany J. Systematic review of the literature informing the systemic lupus erythematosus indicators project: reproductive health care quality indicators. Arthritis Care Res 2011;63:17-30.

17. Campbell S, Griffin DR, Pearce JM, Diaz-Recasens J, Cohen-Overbeek TE, Willson K, et al. New doppler technique for assessing uteroplacental blood flow. Lancet 1983;1:675-7.

18. Demas KL, Costenbader KH. Disparities in lupus care and outcomes. Curr Opin Rheumatol 2009;21:102-9.

19. Moher D, Shamseer L, Clarke M, Ghersi D, Liberati A, Petticrew $\mathrm{M}$, et al. Preferred reporting items for systematic review and meta-analysis protocols (PRISMA-P) 2015 statement. Syst Rev 2015;4:1.

20. Al Arfaj AS, Khalil N. Pregnancy outcome in 396 pregnancies in patients with SLE in Saudi Arabia. Lupus 2010;19:1665-73.

21. Alshohaib S. Outcome of pregnancy in patients with inactive systemic lupus erythromatosus and minimal proteinuria. Saudi J Kidney Dis Transpl 2009;20:802-5.

22. Bertolaccini ML, Mepani K, Sanna G, Hughes GR, Khamashta MA. Factor XII autoantibodies as a novel marker for thrombosis and adverse obstetric history in patients with systemic lupus erythematosus. Ann Rheum Dis 2007;66:533-6.

23. Brucato A, Doria A, Frassi M, Castellino G, Franceschini F, Faden $\mathrm{D}$, et al. Pregnancy outcome in 100 women with autoimmune diseases and anti-Ro/SSA antibodies: a prospective controlled study. Lupus 2002;11:716-21.

24. Clowse ME, Magder LS, Petri M. The clinical utility of measuring complement and anti-dsDNA antibodies during pregnancy in patients with systemic lupus erythematosus. J Rheumatol 2011;38:1012-6

25. Cortés-Hernández J, Ordi-Ros J, Paredes F, Casellas M, Castillo F, Vilardell-Tarres M. Clinical predictors of fetal and maternal outcome in systemic lupus erythematosus: a prospective study of 103 pregnancies. Rheumatology 2002;41:643-50.

26. Daskalakis GJ, Kontessis PS, Papageorgiou IS, Paraskevopoulos AP, Digenis GE, Karaiskakis PT, et al. Lupus nephritis and pregnancy. Hyperten Preg 2009;17:23-30.

27. Derksen RH, Bruinse HW, de Groot PG, Kater L. Pregnancy in systemic lupus erythematosus: a prospective study. Lupus 1994:3:149-55.

28. Farine D, Granovsky-Grisaru S, Ryan G, Seaward PGR, Teoh TG, Laskin $\mathrm{C}$, et al. Umbilical artery blood flow velocity in pregnancies complicated by systemic lupus erythematosus. J Clin Ultrasound 1998;26:379-82.

29. Surita FG, Parpinelli MA, Yonehara E, Krupa F, Cecatti JG. Systemic lupus erythematosus and pregnancy: clinical evolution, maternal and perinatal outcomes and placental findings. Sao Paulo Med J 2007;125:91-5.

30. Gaballa HA, El-Shahawy EE, Atta DS, Gerbash EF. Clinical and serological risk factors of systemic lupus erythematosus outcomes during pregnancy. Egypt Rheumatologist 2012;34:159-65 .

31. Gheita TA, Gamal SM, El-Kattan E. Uterine-umbilical artery Doppler velocimetry and pregnancy outcome in SLE patients: Relation to disease manifestations and activity. Egypt Rheumatologist 2011;33:187-93.

32. Le Thi Huong D, Wechsler B, Vauthier-Brouzes D, Duhaut P, Costedoat N, Andreu MR, et al. The second trimester Doppler ultrasound examination is the best predictor of late pregnancy outcome in systemic lupus erythematosus and/or the antiphospholipid syndrome. Rheumatology 2006;45:332-8.

33. Ideguchi H, Ohno S, Uehara T, Ishigatsubo Y. Pregnancy outcomes in Japanese patients with SLE: retrospective review of 55 pregnancies at a university hospital. Clin Rev Allergy 2013; 44:57-64.

34. Jaeggi E, Laskin C, Hamilton R, Kingdom J, Silverman E. The importance of the level of maternal anti-Ro/SSA antibodies as a prognostic marker of the development of cardiac neonatal lupus erythematosus: a prospective study of 186 antibody-exposed fetuses and infants. J Am Coll Cardiol 2010;55:2778-84.

35. Jara LJ, Pacheco-Reyes H, Medina G, Angeles U, Cruz-Cruz P, Saavedra MA. Prolactin levels are associated with lupus activity, lupus anticoagulant, and poor outcome in pregnancy. Ann N Y Acad Sci 2007;1108:218-26.

36. Mokbel A, Geilan AM, AboElgheit S. Could women with systemic lupus erythematosus (SLE) have successful pregnancy outcomes? Prospective observational study. Egypt Rheumatologist 2013;35:133-9.

37. Moroni G, Quaglini S, Banfi G, Caloni M, Finazzi S, Ambroso G, et al. Pregnancy in lupus nephritis. Am J Kidney Dis 2002;40:713-20.

38. Petri M, Ho AC, Patel J, Demers D, Joseph JM, Goldman D. Elevation of maternal alpha-fetoprotein in systemic lupus erythematosus: a controlled study. J Rheumatol 1995;22:1365-8.

39. Salazar-Páramo M, Jara LJ, Ramos A, Barile L, Machado G, García-De La Torre I. Longitudinal study of antinuclear and anticardiolipin antibodies in pregnant women with systemic lupus erythematosus and antiphospholipid syndrome. Rheumatol Int 2002;22:142-7.

40. Stagnaro-Green A, Akhter E, Yim C, Davies TF, Magder L, Petri M. Thyroid disease in pregnant women with systemic lupus erythematosus: increased preterm delivery. Lupus 2011;20:690-9.

41. Andrade RM, McGwin G Jr., Alarcon GS, Sanchez ML, Bertoli AM, Fernandez M, et al. Predictors of post-partum damage accrual in systemic lupus erythematosus: data from LUMINA, a multiethnic US cohort (XXXVIII). Rheumatology 2006;45:1380-4.

42. Clark CA, Spitzer KA, Nadler JN, Laskin CA. Preterm deliveries in women with systemic lupus erythematosus. J Rheumatol 2003;30:2127-32.

43. Mavragani CP, Dafni UG, Tzioufas AG, Moutsopoulos HM. Pregnancy outcome and anti-Ro/SSA in autoimmune diseases: a retrospective cohort study. Br J Rheumatol 1998;37:740-5.

44. Ogasawara M, Aoki K, Hayashi Y. A prospective study on pregnancy risk of antiphospholipid antibodies in association with systemic lupus erythematosus. J Reprod Immunol 1995;28:159-64.

45. Salomonsson S, Dorner T, Theander E, Bremme K, Larsson P, Wahren-Herlenius M. A serologic marker for fetal risk of congenital heart block. Arthritis Rheum 2002;46:1233-41.

46. Tandon A, Ibanez D, Gladman DD, Urowitz MB. The effect of pregnancy on lupus nephritis. Arthritis Rheum 2004;50:3941-6.

47. Leanos-Miranda A, Cardenas-Mondragon G, Ulloa-Aguirre A, Isordia-Salas I, Parra A, Ramirez-Peredo J. Anti-prolactin autoantibodies in pregnant women with systemic lupus erythematosus: Maternal and fetal outcome. Lupus 2007;16:342-9.

48. Mosca M, Strigini F, Doria A, Pratesi F, Tani C, Iaccarino L, et al. Anti-C1q antibodies in pregnant patients with systemic lupus erythematosus. Clin Exp Rheumatol 2007;25:449-52.

49. Zhao C, Zhao J, Huang Y, Wang Z, Wang H, Zhang H, et al. New-onset systemic lupus erythematosus during pregnancy. Clin Rheumatol 2013;32:815-22.

50. Giancotti A, Spagnuolo A, Bisogni F, D’Ambrosio V, Pasquali G, Panici PB. Pregnancy and systemic lupus erythematosus: role of ultrasound monitoring. Eur J Obstet Gynecol Reprod Biol 2011;154:233-4.

51. Gladman G, Silverman ED, Luy L, Boutin C, Laskin C, Smallhorn JF. Fetal echocardiographic screening of pregnancies of mothers with anti-Ro and/or anti-La antibodies. Am J Perinatol 2002; 19:73-80.

Personal non-commercial use only. The Journal of Rheumatology Copyright $\subset$ 2018 . All rights reserved. 
52. Lockshin MD, Kim M, Laskin CA, Guerra M, Branch DW, Merrill $\mathrm{J}$, et al. Prediction of adverse pregnancy outcome by the presence of lupus anticoagulant, but not anticardiolipin antibody, in patients with antiphospholipid antibodies. Arthritis Rheum 2012;64:2311-8.

53. Spence D, Hornberger L, Hamilton R, Silverman ED. Increased risk of complete congenital heart block in infants born to women with hypothyroidism and anti-Ro and/or anti-La antibodies. J Rheumatol 2006;33:167-70.

54. Ruiz-Irastorza G, Khamashta MA. Evaluation of systemic lupus erythematosus activity during pregnancy. Lupus 2004;13:679-82.
55. Ruiz-Irastorza G, Khamashta MA, Gordon C, Lockshin MD, Johns KR, Sammaritan L, et al. Measuring systemic lupus erythematosus activity during pregnancy: validation of the lupus activity index in pregnancy scale. Arthritis Rheum 2004;51:78-82.

56. Alfirevic Z, Neilson JP. Doppler ultrasonography in high-risk pregnancies: systematic review with meta-analysis. Am J Obstet Gynecol 1995;172:1379-87.

57. Chavez M, Guzman ER, Benito C, Yeo L, Vintzileos A. The clinical significance of absence of end-diastolic velocities of the umbilical artery detected in the severely preterm fetus. Obst Gynecol 2001;97:S45. 